\title{
Enteral Versus Parenteral Nutrition for Postoperative Pancreatic Fistula: A Systematic Review and Meta-analysis
}

\author{
Sandra Lee ${ }^{1+}$, Tyler McKechnie ${ }^{1,2+}$, Yung Lee ${ }^{1,2}$, Kasia Tywonek ${ }^{2}$, Aristithes Doumouras ${ }^{1,2,3}$, \\ Leyo Ruo ${ }^{1,2,4}$, Pablo E. Serrano ${ }^{1,2,4^{*}}$
}

\author{
*Corresponding author: \\ Pablo E. Serrano MD, MPH, FACS \\ Division of Hepatobiliary Surgery \\ Department of Surgery \\ McMaster University. 711 \\ Concession St., Rm B3-161 \\ Hamilton, ON L8V 1 C3 \\ Phone: (905) 521-2100 x43872 \\ Fax: (905) 540-6519 \\ E-mail: serrano@mcmaster.ca \\ †Joint first authors, contributed \\ equally
}

'McMaster University, Hamilton, Ontario, Canada

2Department of Surgery, Division of General Surgery, McMaster University, Hamilton, Ontario, Canada ${ }^{3}$ Division of General Surgery, Department of Surgery, St. Joseph's Healthcare, Hamilton, Ontario, Canada

${ }^{4}$ Division of General Surgery, Department of Surgery, Juravinski Hospital, Hamilton, Ontario, Canada

\section{ABSTRACT}

Background: Postoperative pancreatic fistula (POPF) remains a significant source of morbidity following pancreatic surgery. The most effective feeding route for the conservative management of POPF is currently unknown. We aimed to compare the efficacy of enteral to parenteral nutrition in the rate of POPF closure.

Methods: Medline, EMBASE, CENTRAL, and Web of Science were searched for randomized controlled trials (RCTs) comparing enteral to parenteral nutrition in the conservative management of POPF. Risk of bias was assessed using the Cochrane Risk of Bias Tool. Quality of the evidence was assessed using GRADE. Random-effects meta-analysis was used to estimate time to POPF closure.

Results: From 2,682 citations, three RCTs $(n=167)$ were included (enteral group, $n=85$; parenteral group, $\mathrm{n}=82$ ). Mean time to POPF closure was 3.64 days shorter in the enteral than the parenteral group, which was not statistically significant $(95 \% \mathrm{Cl}-3.22$ to $10.49, \mathrm{P}$ $=0.30$ ). There were no significant differences in postoperative complication rate (OR 1.69, $95 \% \mathrm{Cl} 0.52$ to $5.47, \mathrm{P}=0.38$ ) or length of hospital stay between groups (mean difference: $0.76,95 \% \mathrm{Cl}-9.21$ to $10.74, \mathrm{P}=0.88$ ). According to GRADE, the overall certainty of the evidence for the main outcome was very low.

Conclusions: There was no data to suggest that enteral nutrition is more effective than parenteral nutrition at enhancing the rate of POPF closure. However, the quality of evidence that currently exists is very low. Therefore, the decision between enteral and parenteral nutrition in this setting should still be based on physician preference and patient specific factors.

Key words: hepatobiliary, postoperative pancreatic fistula, fistula, postoperative complication, pancreatioduodenectomy, pancreatectomy

\section{BACKGROUND}

Postoperative pancreatic fistula (POPF) remains a paramount concern for hepatobiliary surgeons, as they constitute a large proportion of potential morbidity following pancreatic surgeries with studies reporting a POPF incidence of $10 \%$ to $40 \%$ (1-4). The International Study Group of Pancreatic 
Surgery (ISGPS) defines POPF as a drain output of any measurable volume of fluid with an amylase level more than three times the upper limit of institutional normal serum amylase activity, associated with a clinically relevant condition directly related to the fistula (5). Classification of POPFs depends on their clinical course and is divided into three separate grades. Grade A POPF, otherwise known as a biochemical leak, has no impact on clinical management, Grade B POPF requires a change in clinical management, and lastly, a Grade C POPF requires significant deviation from the clinical course, such as reoperation, multi-organ failure, and/or mortality attributable to the fistula (5). Of those who develop POPF, around $60 \%$ are clinically relevant, resulting in delayed hospital discharge and additional treatments (6). Fluid and electrolyte imbalance, nutritional depletion, sepsis, abscess and hemorrhage are all possible consequences of POPF (7). Currently, conservative treatment with nutritional supplementation is widely used in the management of POPF (8).

Feeding routes vary and can be widely categorized as enteral or parenteral. Oral feeds, a type of enteral feed, have been demonstrated in randomized controlled trials (RCTs) to be a tolerable and feasible management option for POPF (9). Nonetheless, the intraluminal passage of nutrients through the gastrointestinal tract triggers the release of gastrointestinal hormones, promoting the secretion of pancreatic digestive enzymes (10). It is thought that these enzymes may prolong closure of the fistula (10). As such, the use of enteral jejunal tube feeds has been investigated as they not only avoid pancreatic stimulation, but may also trigger the release of specific gut peptides that inhibit pancreatic enzymes (11). However, the use of enteral feeding tubes is associated with complications such as ileus, aspiration pneumonia, and feeding tube-related complications (i.e. blockage, migration, dislodgement)(12,13). Total parenteral nutrition (TPN) is a means of bypassing these issues entirely (14). Several studies have reported high fistula closure rates with $\operatorname{TPN}(15,16)$, although long-term use of TPN can result in negative functional and morphological gastrointestinal changes $(17,18)$. Overall, all of these methods of feeding are limited by their own sets of complications and currently, there is inconclusive evidence to indicate that one is superior to another.

Despite a growing number of studies comparing enteral and parenteral nutrition, the literature on feeding strategies for treatment and closure of POPF has yet to be comprehensively reviewed and metaanalyzed. As such, the most effective form of postoperative nutrition for promoting the healing and closure of POPF following pancreatic surgery remain unknown. This systematic review and meta-analysis aims to compare the efficacy of enteral (experimental) to parenteral (control) nutrition on the rates of POPF closure, as well as fistula drain output, hospital length of stay (LOS), and other postoperative complications.

\section{MATERIALS AND METHODS}

\section{Search strategy}

The following databases covering the period from database inception through June 2019 were searched: Medline, EMBASE, Cochrane Central Register of Controlled Trials (CENTRAL), and Web of Science. The full search strategy is available in Appendices A-D. The references of included studies were searched manually to ensure that all relevant articles were included. Grey literature was reviewed from the following sources: abstracts from the American Society of Clinical Oncology, Annals of Surgical Oncology, International Society of Gastrointestinal Cancer, International Hepato-Pancreato-Biliary Association, International Association of Pancreatology, American Society of Parenteral and Enteral Nutrition. Lastly, experts in the field were contacted for information regarding potentially relevant studies. This systematic review and meta-analysis is reported in accordance with the Preferred Reporting for Systematic Reviews and MetaAnalyses (PRISMA)(19). The protocol of this study was registered before commencement in the Prospective Register of Systematic Reviews (registration number: CRD42019137522).

\section{Study selection and data extraction}

Articles were eligible for inclusion if they were original work published in peer-reviewed journals comparing enteral and parenteral nutrition in the conservative management of POPF in patients over the age of 18 who underwent pancreatic surgery and reported one of the primary or secondary outcomes. Enteral nutrition (experimental or intervention group) was defined as any form of intraluminal feeding (i.e. oral, nasogastric or nasojejunal tube). Parenteral nutrition (control group) was defined as intravenous feeds, either TPN or peripheral parenteral nutrition (PPN). The definition of POPF was in accordance with the ISGPS(5). Only RCTs were included. Studies were excluded if they were single-armed or if the included patients with distant metastases either on imaging or intraoperatively. Studies were not discriminated based 
on language, geographical area, or operative approach (i.e., laparoscopic vs. open). Studies were excluded if they contained less than 10 eligible patients in each arm. Observational studies, unpublished abstracts, posters, opinions, case reports, systematic reviews, meta-analyses, letters to editors, and editorials were excluded.

Two reviewers independently evaluated and systematically searched titles and abstracts using a standardized, pilot-tested form. Discrepancies that occurred at the title and abstract screening stages were resolved by automatic inclusion. Discrepancies at the full-text stage were resolved by consensus between two reviewers and if disagreement persisted, a third reviewer was consulted. Interobserver agreement was calculated with the Cohen's kappa coefficient for each step of the screening process. Agreement was classified a priori according to the following: $k=0.81$ to 1.0 was almost perfect agreement; $k=0.61$ to 0.80 was substantial agreement; $\mathrm{k}=0.41$ to 0.60 was moderate agreement; and $\mathrm{k}=0.21$ to 0.40 was fair agreement (20).

Two reviewers independently conducted data extraction using a standardized, pilot-tested form designed a priori. Discrepancies were discussed by both reviewers and resolved by consulting original articles and contacting study authors, if necessary. Abstracted data included study characteristics and patient demographics (e.g. author, year of publication, study design, age, sex, body mass index [BMI]), patient comorbidities (e.g. diabetes, hypertension, heart disease, pulmonary disease, liver cirrhosis, peptic ulcer disease [PUD]), operative details (e.g. specific operation, indication for surgery), fistula drainage (e.g. fistula closure, time to closure, length of drain placement, fistula output, fistula grade), and surgical complications (e.g. hemorrhage, delayed gastric emptying [DGE], superficial surgical infection [SSI], intra-abdominal abscess, biliary leak, ileus, wound infection, LOS).

\section{Outcomes assessed}

The primary outcome was time to POPF closure in days. Secondary outcomes included (1) POPF closure within 30 days of fistula onset; (2) POPF output per day (in $\mathrm{mL}$ ), as well as POPF output in the first 48 hours (in $\mathrm{mL}$ ) of initiating nutritional therapy; (3) hospital LOS; and (4) overall postoperative complications (i.e. hemorrhage, SSI, intra-abdominal abscess, ileus, DGE, biliary leak). Postoperative complications were uniformly defined as any clinically significant, documented deviations from the expected postoperative course in patient medical records. For studies that did not report all secondary outcomes, authors were contacted for missing data. If there was no response from authors of the included studies within two weeks, the data was acknowledged as missing.

\section{Risk of Bias Assessment and Quality of the Evidence}

Risk of bias for each included study was assessed using the Cochrane Risk of Bias Tool for RCTs (22). This tool assesses RCTs according to random sequence generation, allocation concealment, selective reporting, blinding of participants and personnel, blinding of outcome assessment, outcome data, and other biases. Good quality evidence has low risk of bias across all domains, fair quality evidence has one category described as having high risk of bias, and poor-quality evidence has two or more categories described as having high or unclear risk of bias. Quality of evidence for estimates derived from meta-analyses was assessed by the Grading of Recommendations, Assessment, Development, and Evaluation (GRADE) approach (23). This approach determines the confidence in the estimate of pooled effects for each individual study outcome. The reported outcomes are analyzed in the individual studies according to risk of bias, inconsistency of results, indirectness of evidence, imprecision, and publication bias.

\section{Statistics}

All statistical analysis and meta-analysis were performed on STATA version 14 (StataCorp, College, TX) and Cochrane Review Manager 5.3 (London, United Kingdom) and verified by a qualified statistician at McMaster University. The threshold for statistical significance was set a priori at a $\mathrm{P}$ value of $<0.05$. We performed pairwise meta-analyses using a DerSimonian and Laird random effects model for all meta-analyzed outcomes. Pooled effect estimates were obtained by calculating the mean difference (MD) in outcomes for continuous variables and risk ratios (RR) for dichotomous variables along with their respective 95\% confidence intervals $(\mathrm{Cl})$ to confirm the effect size estimation. In addition, mean and standard deviation (SD) were estimated for studies that only reported median and interquartile range using Wan et al estimation method (21). Assessment of heterogeneity was completed using the inconsistency $\left(\mathrm{I}^{2}\right)$ statistic. An $1^{2}$ higher than $50 \%$ was considered to represent considerable heterogeneity. For outcomes that are 
unable to be meta-analyzed, a systematic narrative summary of outcomes was provided.

\section{RESULTS}

\section{Study characteristics}

From 2,682 relevant citations, three RCTs met all of the inclusion criteria(24-26). A PRISMA flow diagram of the study selection process is illustrated in fig. 1. Study selection revealed substantial agreement for full-text review (Cohen's kappa $=0.61$ ). Studies were conducted between 2011 and 2015. Two of the included studies were conducted by the same research group, with a focus on different surgical procedures, namely pancreaticoduodenectomy (PD)(26) and distal pancreatectomy (25). Overall, 85 patients were randomized to the enteral nutrition group ( $42.4 \%$ female, mean age 63.1 years, mean BMI 23.3) and 82 patients were randomized to the parenteral nutrition group (39.0\% female, mean age 59.8 years, mean BMI 23.4). In total, 94 patients $(56.3 \%)$ underwent PD, 37 patients $(22.2 \%)$ underwent distal pancreatectomy, and 36 patients (21.5\%) underwent other pancreatic operations (e.g. necrosectomy). The operations were evenly distributed amongst the two groups. The most common indication for operation was pancreatic adenocarcinoma $(n=57$, $34.1 \%$ ), followed by ampullary neoplasms ( $n=24$, $14.4 \%)$, intraductal pancreatic mucinous neoplasm (IMPN) $(n=14,8.4 \%)$, and cholangiocarcinoma $(n=11$,
6.6\%). Detailed description of study characteristics, operative characteristics, disease characteristics, and comorbidities are reported in table 1.

\section{Fistula closure}

All three studies reported time to closure in days ( $\mathrm{n}$ $=167$ ). Klek et al. reported median fistula duration in days, while Fujii et al. reported median length of fistula drain placement (table 2). In the study by Klek et al. fistula closure was defined as a drain output of less than $10 \mathrm{~mL}$ per 24 hours(24). In the remaining two studies by Fujii et al. fistula closure was defined by removal of the fistula drain, which occurred when output was less than $20 \mathrm{~mL}$ per 24 hours $(25,26)$. Patients in the included studies did not receive any other therapies aimed at hastening fistula closure (e.g. proton pump inhibitors, somatostatin analogues). The median fistula duration reported by Klek et al. was 37.5 days (IQR 25.0-61.7) in the enteral group and 43.5 days (IQR 28.7-54.0) in the parenteral group. The median length of drain placement reported in the studies by Fujii et al. was 22 days (range $=6$ to 80 days) in the enteral group and 21.2 days (range $=7$ to 112 days) in the parenteral group. These outcomes were pooled for effects estimate (fig. 2). The mean time to POPF closure was 3.46 days shorter in the enteral group than the parenteral group $(95 \% \mathrm{Cl}-1.39$ to 8.31 , $P=0.30)$. The degree of heterogeneity was likely insignificant $\left(I^{2}=39 \%\right)$.
Figure 1 - PRISMA Diagram. Transparent reporting of systematic reviews and meta-analysis flow diagram showing initial search strategy results to included studies

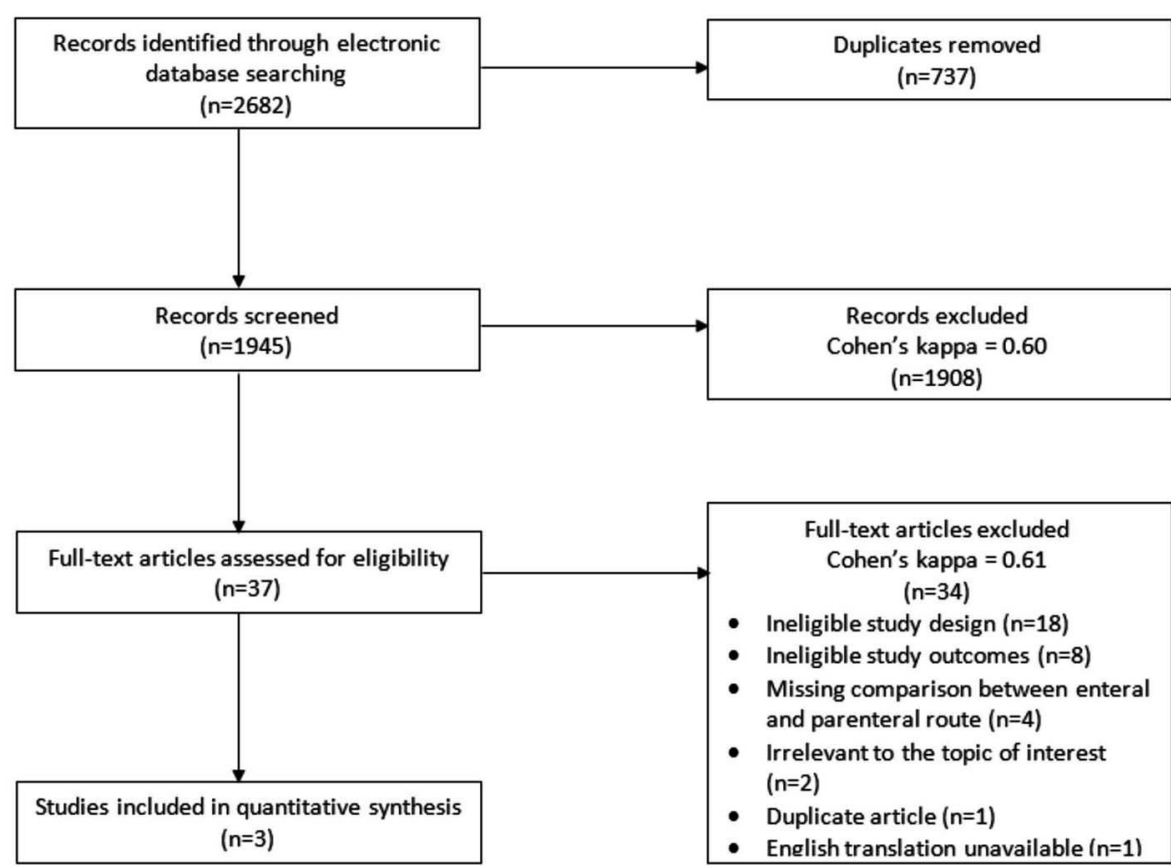


Table 1 - Study Characteristics (N - number of patients, y - years, BMI - body mass index, kg - kilograms, $\mathrm{m}$ - meters,

PD - pancreaticoduodenectomy, IPMN - intraductal papillary mucinous neoplasm, HTN - hypertension, PUD - peptic ulcer disease)

\begin{tabular}{|c|c|c|c|c|c|c|c|c|c|c|c|c|c|c|}
\hline \multirow[t]{2}{*}{ Author } & \multirow[t]{2}{*}{ Arm } & \multirow[t]{2}{*}{$\mathrm{N}$} & \multirow{2}{*}{$\begin{array}{c}\% \\
\text { Female }\end{array}$} & \multirow{2}{*}{$\begin{array}{c}\text { Age } \\
(y, \\
\text { median) }\end{array}$} & \multirow{2}{*}{$\begin{array}{c}\text { BMI } \\
\left(\mathrm{kg} / \mathrm{m}^{2}\right. \\
\text { median) }\end{array}$} & \multicolumn{3}{|c|}{ Operation } & \multicolumn{5}{|c|}{ Indication for Operation } & \multirow{2}{*}{$\begin{array}{c}\text { Comorbidities } \\
(\%)\end{array}$} \\
\hline & & & & & & $\begin{array}{l}\text { PD } \\
(\%)\end{array}$ & $\begin{array}{c}\text { Distal } \\
\text { Pancreatectomy } \\
(\%)\end{array}$ & $\begin{array}{c}\text { Other } \\
(\%)\end{array}$ & $\begin{array}{l}\text { Pancreatic } \\
\text { Adeno- } \\
\text { carcinoma } \\
(\%)\end{array}$ & $\begin{array}{l}\text { Cholangio- } \\
\text { carcinoma } \\
(\%)\end{array}$ & $\begin{array}{l}\text { Ampullary } \\
\text { neoplasm } \\
(\%)\end{array}$ & $\begin{array}{l}\text { IPMN } \\
(\%)\end{array}$ & $\begin{array}{l}\text { Other } \\
(\%)\end{array}$ & \\
\hline \multirow[t]{2}{*}{$\begin{array}{l}\text { Klek, } \\
2011\end{array}$} & Parenteral & 38 & 44.7 & 55 & 23.4 & $\begin{array}{l}14 \\
(36.8)\end{array}$ & $4(10.5)$ & $\begin{array}{l}16 \\
(52.6)\end{array}$ & $12(31.6)$ & - & $5(13.2)$ & - & $\begin{array}{l}21 \\
(55.3)\end{array}$ & $\begin{array}{l}\text { Diabetes - } 11 \\
\text { (28.9) } \\
\text { Heart disease - } \\
13 \text { (34.2) } \\
\text { HTN - } 14 \text { (36.8) } \\
\text { Pulmonary } \\
\text { disease - } 3(7.9) \\
\text { Liver cirrhosis - } 0\end{array}$ \\
\hline & Enteral & 40 & 45.0 & 60.5 & 24.9 & $\begin{array}{l}21 \\
(52.5)\end{array}$ & $3(7.5)$ & $\begin{array}{l}16 \\
(40.0)\end{array}$ & $15(37.5)$ & - & $9(22.5)$ & - & $\begin{array}{l}16 \\
(40.0)\end{array}$ & $\begin{array}{l}\text { Diabetes - } 8 \\
(20.0) \\
\text { Heart disease - } \\
16(40.0) \\
\text { HTN - } 21 \text { (52.5) } \\
\text { Pulmonary } \\
\text { disease - } 4(10.0) \\
\text { Liver cirrhosis - } 1 \\
(2.5)\end{array}$ \\
\hline \multirow[t]{2}{*}{$\begin{array}{l}\text { Fujii } \\
\text { (DP), } \\
2015\end{array}$} & Parenteral & 15 & 33.3 & 62 & 23.6 & - & $15(100)$ & - & $11(73.3)$ & - & - & $\begin{array}{l}1 \\
(6.67)\end{array}$ & $\begin{array}{l}3 \\
(20.0)\end{array}$ & $\begin{array}{l}\text { Diabetes - } 5 \\
(33.3) \\
\text { Heart disease - } 2 \\
(13.3) \\
\text { HTN - } 0 \\
\text { Pulmonary } \\
\text { disease - } 1(6.7) \\
\text { PUD - } 0\end{array}$ \\
\hline & Enteral & 15 & 40.0 & 67.5 & 22.3 & $\cdot$ & $15(100)$ & - & $7(46.7)$ & - & - & $\begin{array}{l}3 \\
(20.0)\end{array}$ & $\begin{array}{l}5 \\
(33.3)\end{array}$ & $\begin{array}{l}\text { Diabetes - } 2 \\
(13.3) \\
\text { Heart disease - } 1 \\
\text { (6.7) } \\
\text { HTN - } 2 \text { (13.3) } \\
\text { Pulmonary } \\
\text { disease - } 1(6.7) \\
\text { PUD - } 2 \text { (13.3) }\end{array}$ \\
\hline \multirow[t]{2}{*}{$\begin{array}{l}\text { Fujii } \\
\text { (PD), } \\
2015\end{array}$} & Parenteral & 29 & 34.5 & 65 & 23.3 & $\begin{array}{l}29 \\
(100)\end{array}$ & - & - & $9(31.0)$ & $5(17.2)$ & $6(20.7)$ & $\begin{array}{l}7 \\
(24.1)\end{array}$ & $\begin{array}{l}2 \\
(6.9)\end{array}$ & $\begin{array}{l}\text { Diabetes - } 5 \\
(17.2) \\
\text { Heart disease - } 2 \\
(6.9) \\
\text { HTN - } 12 \text { (41.4) } \\
\text { Pulmonary } \\
\text { disease - } 0 \\
\text { PUD - } 1(3.4)\end{array}$ \\
\hline & Enteral & 30 & 40.0 & 64.5 & 21.7 & $\begin{array}{l}30 \\
(100)\end{array}$ & - & - & $13(43.3)$ & $6(20.0)$ & $4(13.3)$ & $\begin{array}{l}3 \\
(10.0)\end{array}$ & $\begin{array}{l}4 \\
(13.3)\end{array}$ & $\begin{array}{l}\text { Diabetes - } 7 \\
(23.3) \\
\text { Heart disease - } 3 \\
(10.0) \\
\text { HTN - } 10 \text { (33.3) } \\
\text { Pulmonary } \\
\text { disease - } 2 \text { (6.7) } \\
\text { PUD - } 4 \text { (13.3) }\end{array}$ \\
\hline
\end{tabular}


Table 2 - Post-operative pancreatic fistula drain-related outcomes from included studies ( $\mathrm{N}$ - number, IQR - inter-quartile range, POPF - post-operative pancreatic fistula, $\mathrm{d}$ - days, $\mathrm{Cl}$ - confidence interval, $\mathrm{mL}$ - millilitres, POD - post-operative day, LOS - length of stay)

\begin{tabular}{|c|c|c|c|c|c|c|c|c|c|c|}
\hline \multirow[t]{2}{*}{ Author } & \multirow[t]{2}{*}{ Arm } & \multirow[t]{2}{*}{ N } & \multirow{2}{*}{$\begin{array}{l}\text { N POPF } \\
\text { Closure by } \\
\text { 30d (\%) }\end{array}$} & \multirow{2}{*}{$\begin{array}{l}\text { Fistula duration } \\
\qquad \text { (d, IQR) }\end{array}$} & \multirow{2}{*}{$\begin{array}{l}\text { Median length of } \\
\text { drain placement } \\
\text { (d, range) }\end{array}$} & \multicolumn{4}{|c|}{ Median POPF Drain Output (mL, range) } & \multirow{2}{*}{$\begin{array}{l}\text { Median LOS } \\
\text { (d, range) }\end{array}$} \\
\hline & & & & & & POD 1 & POD 3 & POD 5 & POD 7 & \\
\hline \multirow{2}{*}{$\begin{array}{l}\text { Klek, } \\
2011\end{array}$} & Parenteral & 38 & $14(36.8)$ & $43.5(28.7-54.0)$ & - & - & - & - & - & - \\
\hline & Enteral & 40 & $24(60.0)$ & $37.5(25.0-61.7)$ & - & - & - & - & - & - \\
\hline \multirow{2}{*}{$\begin{array}{l}\text { Fujii } \\
\text { (DP), } \\
2015\end{array}$} & Parenteral & 15 & - & - & $12(7-112)$ & $56(14-250)$ & $10(1-270)$ & $5(1-50)$ & $5(1-35)$ & $26(15-119)$ \\
\hline & Enteral & 15 & - & - & $12(6-58)$ & $46(5-120)$ & $10(1-60)$ & $4(1-60)$ & $5(1-35)$ & $24(12-91)$ \\
\hline Fujii & Parenteral & 29 & - & - & $26(7-70)$ & $213(51-600)$ & $44(2-187)$ & $21(1-221)$ & $15(0-225)$ & $29(17-78)$ \\
\hline $\begin{array}{l}\text { (PD), } \\
2015\end{array}$ & Enteral & 30 & - & - & $27(7-80)$ & $170(52-385)$ & $32.5(2-167)$ & $10(2-845)$ & $10.5(2-865)$ & $29.5(16-88)$ \\
\hline
\end{tabular}

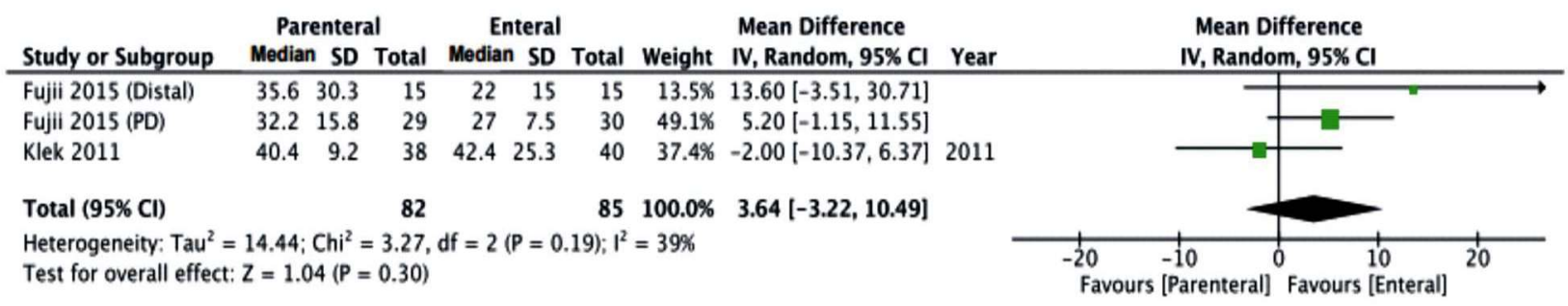

Figure 2 - Time to Fistula Closure. Comparison of time to fistula closure in days in parenteral and control groups in individual studies and the pooled time to closure. Pairwise meta-analysis was performed using a DerSimonian and Laird random effects model. Mean difference is shown with $95 \%$ confidence intervals

\section{Fistula output}

The two studies by Fujii et al. reported fistula output by postoperative day (POD) (table 2). There were no significant differences between POPF drain output between parenteral and enteral groups in either study. POPF drain output decreased over time. In the enteral group, the mean POPF drain output was $128.7 \mathrm{~mL}$ (range $=5$ to $385 \mathrm{~mL}$ ) on POD 1, $25.0 \mathrm{~mL}$ (range $=1$ to $167 \mathrm{~mL}$ ) on POD $3,8.0 \mathrm{~mL}$ (range $=1$ to $845 \mathrm{~mL}$ ) on POD 5 , and $8.7 \mathrm{~mL}$ (range $=1$ to $865 \mathrm{~mL}$ ) on POD 7. In the parenteral groups, the mean POPF drain output was $159.5 \mathrm{~mL}$ (range $=14$ to $600 \mathrm{~mL}$ ) on POD $1,32.4 \mathrm{~mL}$ (range $=1$ to $270 \mathrm{~mL}$ ) on POD 3, $15.5 \mathrm{~mL}$ (range $=1$ to $221 \mathrm{~mL}$ ) on POD 5, and $11.2 \mathrm{~mL}$ (range $=0$ to $225 \mathrm{~mL}$ ) on POD 7. Klek et al. did not stratify drain output by POD, but rather reported median daily POPF drain output. The median outputs were $130.0 \mathrm{~mL}$ (IQR 72.5125.0) and $172.5 \mathrm{~mL}$ (IQR 95.0-167.5) in the enteral and parenteral groups, respectively.

\section{Complications}

Two of the included studies reported overall postoperative complications (table 3$)(25,26)$. There was no significant difference in postoperative complication
Table 3 - Complication rates from included studies (N, number; SSI, surgical site infection; DGE, delayed gastric emptying)

\begin{tabular}{|c|c|c|c|c|c|c|c|}
\hline Author & Arm & $\mathbf{N}$ & SSI (\%) & $\begin{array}{l}\text { Intra- } \\
\text { abdominal } \\
\text { abscess } \\
\text { (\%) }\end{array}$ & Ileus (\%) & DGE (\%) & $\begin{array}{l}\text { Biliary } \\
\text { leak (\%) }\end{array}$ \\
\hline \multirow{2}{*}{$\begin{array}{l}\text { Klek, } \\
2011\end{array}$} & Parenteral & 38 & - & - & - & - & - \\
\hline & Enteral & 40 & - & - & - & - & - \\
\hline \multirow{2}{*}{$\begin{array}{l}\text { Fujii } \\
\text { (DP), } \\
2015\end{array}$} & Parenteral & 15 & $1(6.67)$ & $3(20.0)$ & $1(6.67)$ & $1(6.67)$ & 0 \\
\hline & Enteral & 15 & $1(6.67)$ & $3(20.0)$ & 0 & 0 & 0 \\
\hline \multirow{2}{*}{$\begin{array}{l}\text { Fujii } \\
\text { (PD), } \\
2015\end{array}$} & Parenteral & 29 & $1(3.44)$ & $2(6.90)$ & 0 & 0 & 0 \\
\hline & Enteral & 30 & $1(3.33)$ & $2(6.67)$ & 0 & 0 & 0 \\
\hline
\end{tabular}


rates between the enteral and parenteral groups in either study. Overall morbidity in the Fujii et al. study evaluating patients undergoing DP was 40\% (6/15 patients) in the parenteral group and $26.7 \%(4 / 15$ patients) in the enteral group (25). In both groups, one patient developed a surgical site infection (SSI) (6.67\%) and three patients developed intra-abdominal abscesses (20.0\%). Only patients managed with parenteral nutrition developed paralytic ileus (6.7\%, 1/15 patients) and delayed gastric emptying (DGE) $(6.7 \%, 1 / 15$ patients). In the Fujii et al. study evaluating patients undergoing PD, the overall morbidity rate was $10.3 \%$ (3/29 patients) in the parenteral group and $10.0 \%$ ( $3 / 30$ patients) in the enteral group (26). In both groups, one patient developed an SSI (3.44\%) and two patients developed an intra-abdominal abscess (6.90\%). In both studies, the postoperative LOS was similar between the enteral and parenteral groups. In DP patients, the median LOS was 26 days (range $=15$ to 119 days) in the parenteral group and 24 days (range $=12$ to 91 days) in the enteral group (25). In PD patients, the median LOS was 29 days (range $=17$ to 78 days) in the parenteral group and 29.5 days (range $=16$ to 88 days) in the enteral group(26).

\section{Risk of bias}

The risk of bias from randomization and availability of outcome data was low across all studies (supplementary figs. 1 and 2). All of the included studies utilized concealed, random sequence allocation to distribute patients amongst the two arms of their respective studies. Two of the included studies were at high risk of bias due to assignment as they were not blinded trials $(25,26)$. There was some concern for bias due to method of measuring the outcome across all trials, as outcome assessors were aware of the intervention received. Complete adherence to assigned intervention was not achieved across all studies, yet appropriate analysis was

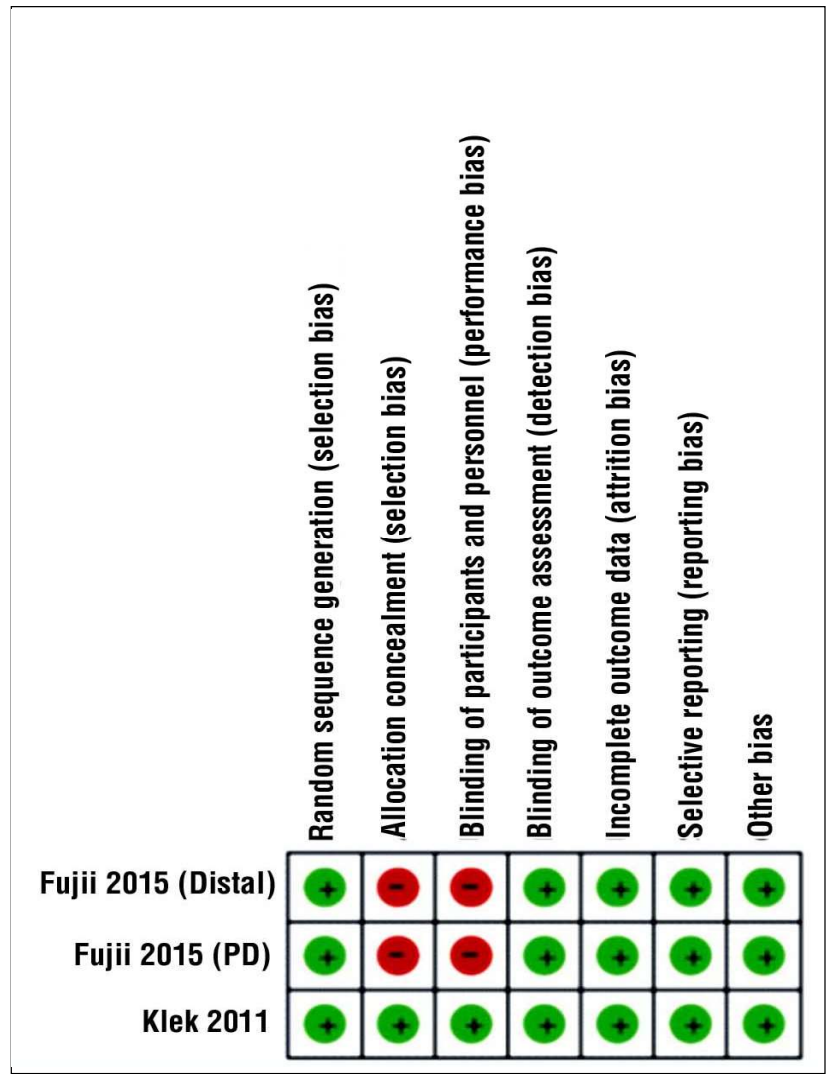

Supplementary Figure 1 - Cochrane Risk of Bias Tool Summary. Risk of bias for each of the included studies. + represents low risk of bias and - represents high risk of bias

used to estimate the effect of adherence. Both Fujii et al. studies utilized parenteral nutrition as a supplement in patients receiving enteral nutrition in order to ensure equal caloric consumption between groups $(25,26)$. As such, two of the studies were associated with some concern for bias with regards to adherence, while one was associated with low risk of bias. Overall, the risk of bias in the included studies was relatively low. One study scored 'low risk of bias' (24), while the other two studies scored 'some concern for bias' $(25,26)$.
Supplementary Figure 2 Cochrane Risk of Bias Tool Graph. Pooled risk of bias according to the separate categories of bias outlined by the Cochrane Risk of Bias Tool. Green represents low risk of bias and red represents high risk of bias

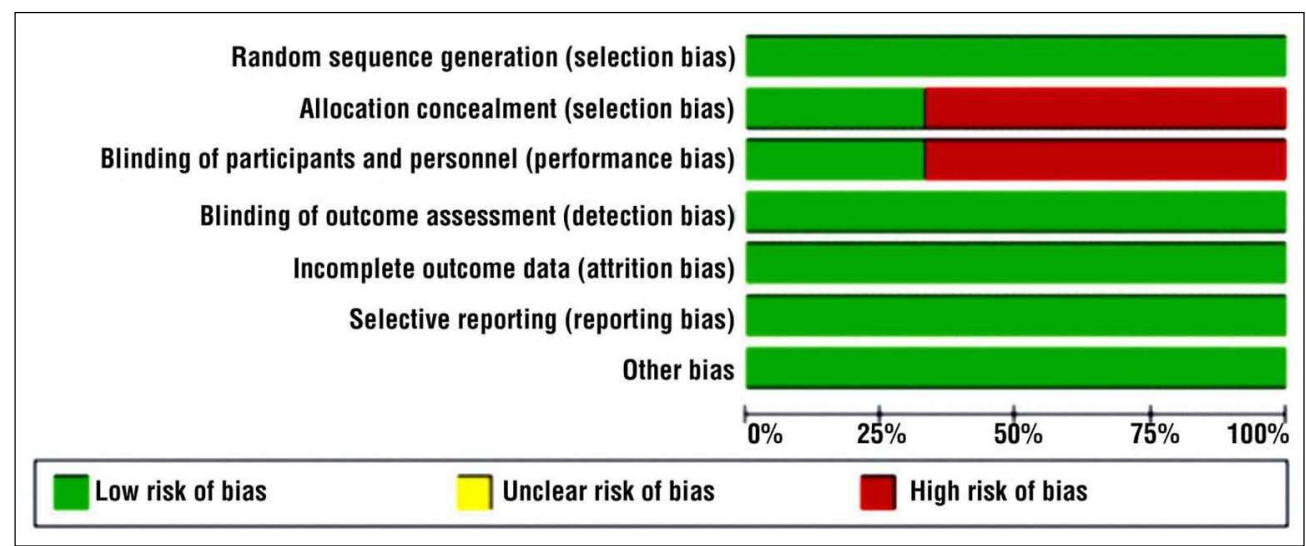




\section{Quality of evidence}

The overall certainty of the evidence for the main outcome of the present study, time to fistula closure in days, was very low (table 4). There were only three RCTs in the present study that reported the main outcome, raising serious concern with regards to imprecision of measurement and publication bias. Given that there were also slightly different criteria for the main outcome of interest, the risk of bias due to indirect comparison between study groups was serious. All three RCTs were powered to detect differences in time to fistula closure or drain removal in days. There were no serious concerns for bias in the included studies or inconsistency between studies impacting main outcome meta-analysis.

\section{DISCUSSION}

Most pancreatic fistulas are managed conservatively, which includes adequate source control (i.e. drainage) and nutritional support $(15,16)$. The optimal method of delivering such nutrition continues to be a topic of debate. As such, the present systematic review and meta-analysis investigated the effect of enteral nutrition compared to parenteral nutrition in the rate of fistula closure. Pooled data in the current study suggested that POPF closure occurred an average of 3.64 days sooner in patients receiving enteral nutrition compared to those receiving parenteral nutrition, however these results were not statistically significant $(P=0.30)$. Data points for other secondary outcomes, such as postoperative complications, LOS, drain output and amylase levels in fistula drainage were not available across all three studies and thus were described narratively.

The risk of bias for each independent study, according to the Cochrane Risk of Bias Tool was low. Random sequence allocation, blinding outcome assessment, and analysis for measuring effect of adherence were all used appropriately to minimize risk of bias. However, while none of the included studies were at extremely high risk of bias, an important confounding variable that was not accounted for in any of the included studies was the proportion of patients with high fistula output at the time of diagnosis. High fistula output ( $>200 \mathrm{~mL} /$ day) at the time of diagnosis has been associated with delayed POPF closure (24). Nonetheless, due to randomization, there were no significant differences between the enteral and parenteral nutrition groups at baseline in any of the included studies, thus reducing the likelihood that other possible confounding factors impacted the results of the present study. Despite the low risk of bias of each independent study, the quality of the evidence across all studies was low; largely due to imprecision secondary to insufficient sample size despite pooling. Currently, only three RCTs comparing enteral and parenteral feeds exist in the literature (24-26). This also raises significant concern for publication bias. Additionally, given the heterogeneity in definitions of duration of POPF, the possibility of indirect comparison further downgrades the quality of evidence. However, other factors in the design of the included studies were similar, thus validating this indirect comparison (23). Moreover, all three of the included studies were adequately powered to detect a clinically relevant difference in the primary outcome (24-26). As such, the pooled estimate would

Table 4 - Grading of Recommendations, Assessment, Development, and Evaluation (GRADE) of meta-analyzed outcomes

\begin{tabular}{|c|c|c|c|c|c|c|c|c|}
\hline \multicolumn{7}{|c|}{ Certainty assessment } & \multicolumn{2}{|c|}{ Summary of findings } \\
\hline $\begin{array}{c}\text { of } \\
\text { participants }\end{array}$ & $\begin{array}{c}\text { Risk of } \\
\text { bias }\end{array}$ & Inconsistency & Indirectness & Imprecision & $\begin{array}{c}\text { Publication } \\
\text { bias }\end{array}$ & $\begin{array}{l}\text { Overall } \\
\text { certainty of } \\
\text { evidence }\end{array}$ & $\begin{array}{c}\text { Pooled } \\
\text { effect size } \\
(95 \% \mathrm{CI})\end{array}$ & Anticipated effects \\
\hline \multicolumn{9}{|c|}{ Time to fistula closure (days) } \\
\hline $\begin{array}{c}167 \\
\text { (3 RCTs) }\end{array}$ & $\begin{array}{c}\text { not } \\
\text { serious }\end{array}$ & not serious $^{2}$ & serious $^{3}$ & serious $^{4}$ & serious $^{5}$ & VERY LOW & $\begin{array}{c}\text { MD } 3.46, \\
95 \% \text { CI - } \\
1.39 \text { to } \\
8.31 \\
\mathrm{P}=0.30 \\
\mathrm{I}^{2}=39 \%\end{array}$ & $\begin{array}{l}\text { No difference between } \\
\text { enteral nutrition and } \\
\text { parenteral nutrition in } \\
\text { time to fistula closure. }\end{array}$ \\
\hline
\end{tabular}

1. All of the trials included had adequate randomization, low attrition bias, and low reporting bias. However, the majority of studies did not blind participants, healthcare providers, and outcome assessors due to the nature of the comparison including the use of intravenous or tube feeds. Nonetheless, blinding is less important for the outcomes analyzed in the present meta-analysis, as the definitions were uniform and the measurements were objective. Therefore, the quality of the evidence was not downgraded. 2. ${ }^{2}<40 \%$ with similar point estimates and overlapping confidence intervals. 3. Although a direct comparison was made between enteral and parenteral groups, downgraded by one point because studies used slightly different criteria for the outcome of interest (i.e. fistula closure was defined as $<10 \mathrm{~mL}$ or $<20 \mathrm{~mL}$ across studies). 4. Downgraded two points due to low number $(n=3)$ of randomized controlled trials present on this topic, wide confidence intervals that overlap no effect as well as both enteral and parenteral nutrition. This suggests studies with larger sample sizes are needed.5. Downgraded one point due to low number $(n=3)$ of randomized controlled trials present on this topic, with a small number of patients in either interventional arm ( $n=82$ in parenteral group; $n=85$ in enteral group). 
Appendix A - OVID Medline Epub Ahead of Print, In-Process \& 0ther Non-Indexed Citations, Ovid MEDLINE $®$ Daily and Ovid MEDLINE $®$ search strategy <1946 to 22 May 2019>

1 exp Parenteral nutrition/

2 parenter* nutrition.mp.

3 parenter* route.mp.

4 TPN.mp.

5 total nutrient admixture.mp.

6 central venous nutrition.mp.

7 parenter* feeding.mp.

8 nil per os.mp

9 NPO.mp.

10 fasting.mp.

11 fluid therapy.mp.

12 intravenous feeding.mp.

13 intravenous nutrition.mp

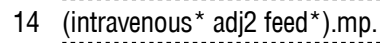

15 (intravenous* adj2 nutrition*).mp.

16 or/1-15

17 exp Enteral nutrition/

18 exp Eating/

19 oral feeding.mp.

20 oral diet.mp.

21 peroral diet.mp.

22 peroral feeding.mp.

23 enter $^{*}$ feeding.mp.

24 enter $^{*}$ nutrition.mp.

25 enter $^{\star}$ route.mp.

26 oral food intake.mp.

27 dietary intake.mp.

28 (feeding adj tube ${ }^{\star}$ ).mp.

29 gastric feeding.mp.

30 (jejunostomy and (tube ${ }^{*}$ or feed ${ }^{*}$ or tube feed $\left.{ }^{*}\right)$ ).mp.

31 (nasojejunal and $\left(\right.$ tube ${ }^{*}$ or feed ${ }^{*}$ or tube feed $)$ ).mp

32 (nasogastric and (tube* or feed ${ }^{\star}$ or tube feed $\left.{ }^{\star}\right)$ ).mp.

33 (gastrojejunostomy and (tube ${ }^{\star}$ or feed ${ }^{\star}$ or tube feed $\left.{ }^{*}\right)$ ).mp

34 (percutaneous endoscopic gastrostomy and (tube* or feed ${ }^{*}$ or tube feed $\left.\left.^{*}\right)\right) \cdot \mathrm{mp}$.

35 (PEG and (tube ${ }^{*}$ or line $\left.{ }^{*}\right)$ ).mp.

36 (percutaneous endoscopic jejunostomy and (tube ${ }^{\star}$ or feed ${ }^{\star}$ or tube feed*)).mp

37 (JEJ and (tube ${ }^{\star}$ or line $\left.\left.{ }^{\star}\right)\right) . \mathrm{mp}$.

38 (radiologically inserted gastrostomy and (tube $^{*}$ or feed ${ }^{*}$ or tube feed $\left.\left.^{\star}\right)\right) . \mathrm{mp}$.

39 (RIG and (tube* or line $\left.{ }^{\star}\right)$ ).mp.

40 (percutaneous transperitoneal jejunostomy and (tube* or feed* or tube feed $\left.\left.^{*}\right)\right) \cdot \mathrm{mp}$.

have been powered to detect a difference between enteral and parenteral nutrition if one existed. Klek et al. powered their RCT to determine whether a difference existed in POPF closure rate within 30 days of index operation, therefore it is likely that the lack of statistically significant difference between enteral and parenteral groups in the present study persists to at least 30 days postoperatively.
41 (percutaneous transperitoneal gastrojejunostomy and (tube ${ }^{\star}$ or feed ${ }^{\star}$ or tube feed*)).mp.

42 or $17-41$

43 exp Nutritional support/

$44{\text { nutrition* }{ }^{*} \text { support }}^{\star} . \mathrm{mp}$

45 nutrition* manag $^{\star} . \mathrm{mp}$.

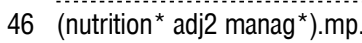

47 artificial nutrition.mp.

48 artificial feeding.mp.

49 nutrition* supplement* $^{*} . \mathrm{mp}$

50 (nutrition $^{*}$ adj2 supplement ${ }^{\star}$ ).mp.

51 postoperative nutrition.mp.

52 perioperative nutrition.mp

53 or $/ 43-52$

54 exp Fistula/

55 exp Digestive system fistula

56 fistula*.mp.

57 fistuliz*mp.

58 fistulis*.mp

59 leak*.mp.

60 or/54-59

61 Pancreatectomy/

62 Pancreaticoduodenectomy/

63 Pancreaticojejunostomy/

64 pancreatectomy.mp.

65 hemipancreatectomy.mp.

66 pancreaticoduodenectomy.mp

67 whipple.mp.

68 whipple procedure ${ }^{\star} . \mathrm{mp}$

69 whipple surgery.mp.

70 distal pancreaticoduodenectomy.mp.

71 pancreaticojejunostomy.mp.

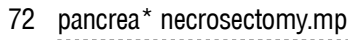

73 pancrea* $^{*}$ surger $^{*} . \mathrm{mp}$

74 or pancrea* procedure $^{*} . \mathrm{mp}$.

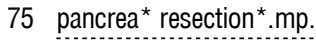

76 (pancrea* $^{\star}$ adj2 surger ${ }^{\star}$ ).mp

77 (pancrea* $^{*}$ adj2 procedure $\left.{ }^{\star}\right) \cdot m p$

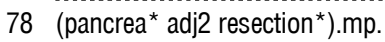

79 Pancreas/

80 pancrea*.mp.

81 or $/ 61-80$

82 (16 or 42 or 53 ) and 60 and 81

This study has other important limitations. Firstly, the definition of fistula closure varied between studies. Klek et al. described fistula closure as fistula output of less than $10 \mathrm{~mL}$ per 24 hours, while both trials from Fujii et al. reported time until drain removal which the current study equated to the duration of a fistula (2426). The criteria to remove the drain was a fistula output of less than $20 \mathrm{~mL}$ per 24 hours, as such, time to 
Appendix B - Embase search strategy $<1974$ to 22 May 2019>

1 exp Parenteral nutrition/

2 exp Fluid therapy/

3 parenter ${ }^{*}$ nutrition.mp

4 parenter $^{*}$ route.mp.

5 TPN.mp

6 total nutrient admixture.mp

7 central venous nutrition.mp.

8 parenter* feeding.mp.

9 nil per os.mp.

10 NPO.mp

11 fasting.mp.

12 fluid therapy.mp.

13 intravenous feeding.mp

14 intravenous nutrition.mp

15 (intravenous $^{*}$ adj2 feed ${ }^{*}$.mp.

16 (intravenous $^{*}$ adj2 nutrition ${ }^{\star}$ ).mp

17 or/1-16

18 exp Enteric feeding/

19 Eating/

20 oral feeding.mp.

21 oral diet.mp.

22 peroral diet.mp.

23 peroral feeding.mp.

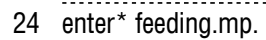

25 enter $^{\star}$ nutrition.mp.

26 enter* route.mp.

27 oral food intake.mp.

28 dietary intake.mp.

29 (feeding adj tube ${ }^{\star}$ ).mp

30 gastric feeding.mp.

31 (jejunostomy and (tube* or feed ${ }^{*}$ or tube feed $\left.{ }^{*}\right)$ ).mp.

32 (nasojejunal and (tube ${ }^{\star}$ or feed ${ }^{\star}$ or tube feed $\left.{ }^{*}\right)$ ).mp.

33 (nasogastric and $\left(\right.$ tube ${ }^{*}$ or feed ${ }^{*}$ or tube feed $\left.{ }^{*}\right)$ ).mp

34 (gastrojejunostomy and (tube ${ }^{*}$ or feed ${ }^{*}$ or tube feed $\left.{ }^{*}\right)$ ).mp

35 (percutaneous endoscopic gastrostomy and (tube ${ }^{\star}$ or feed ${ }^{\star}$ or tube feed $\left.\left.^{*}\right)\right) \cdot \mathrm{mp}$.

36 (PEG and (tube or line $\left.\left.^{\star}\right)\right)$.mp.

37 (percutaneous endoscopic jejunostomy and (tube ${ }^{\star}$ or feed ${ }^{\star}$ or tube feed $\left.\left.^{*}\right)\right) \cdot \mathrm{mp}$.

38 (JEJ and (tube ${ }^{\star}$ or line $\left.{ }^{*}\right)$ ).mp.

39 (radiologically inserted gastrostomy and (tube ${ }^{*}$ or feed ${ }^{*}$ or tube feed $\left.^{*}\right)$ ).mp.

40 (RIG and (tube* or line $\left.\left.{ }^{\star}\right)\right) \cdot \mathrm{mp}$.

41 (percutaneous transperitoneal jejunostomy and (tube ${ }^{\star}$ or feed ${ }^{\star}$ or tube feed $\left.\left.^{\star}\right)\right)$.mp.

42 (percutaneous transperitoneal gastrojejunostomy and (tube ${ }^{\star}$ or feed ${ }^{\star}$ or tube feed $\left.\left.{ }^{\star}\right)\right) \cdot \mathrm{mp}$.

fistula closure may be underestimated in the trials conducted by Fujii and colleagues as compared to the trial performed by Klek and colleagues. Secondly, the small patient samples in the included studies only amounted to a total of 167 patients included in the pooled estimates. Thirdly, differences in the types of pancreatic operation, composition of enteral or parenteral feeds, and time before nutritional treatment
43 or $/ 18-42$

44 exp Nutritional support/

45 exp Artificial feeding/

46 nutrition* $^{*}$ support*.mp.

47 nutrition* manag $^{*} . \mathrm{mp}$.

48 (nutrition* adj2 manag*).mp.

49 artificial nutrition.mp.

50 artificial feeding .mp.

$51{\text { nutrition* }{ }^{*} \text { supplement }}^{*} . \mathrm{mp}$

52 (nutrition $^{*}$ adj2 supplement ${ }^{\star}$ ).mp

53 postoperative nutrition.mp.

54 perioperative nutrition.mp.

55 or/ $/ 44-54$

56 exp Fistula/

57 exp Digestive system fistula/

58 fistula*.mp.

59 fistuliz*.mp.

60 fistulis*.mp.

61 leak*.mp.

62 or/56-61

63 exp Pancreas surgery/

64 exp Pancreatectomy/

65 pancreaticoduodenectomy/

66 pancreaticojejunostomy/

67 pancreatectomy.mp.

68 distal pancreatectomy.mp.

69 total pancreatectomy.mp.

70 hemipancreatectomy.mp.

71 pancreaticoduodenectomy.mp

72 whipple.mp.

73 whipple procedure ${ }^{*} . \mathrm{mp}$

74 whipple surgery.mp.

75 distal pancreaticoduodenectomy.mp.

76 pancreaticojejunostomy.mp.

77 pancrea* necrosectomy.mp

78 pancrea* surger*.mp.

79 pancrea* $^{*}$ procedure*.mp.

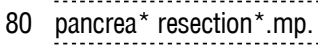

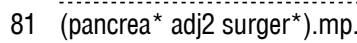

82 (pancrea* $^{*}$ adj2 procedure $\left.{ }^{\star}\right) \cdot m p$

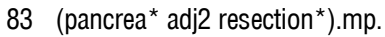

84 Pancreas/

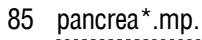

86 or $/ 63-85$

87 (17 or 43 or 55 ) and 55 and 62

initiation among studies are all factors that may have contributed to significant clinical heterogeneity. Heterogeneity in reporting of secondary outcomes, such as drain output and amylase levels in fistula drainage, were also a limitation to the present study and precluded the inclusion of this data in the metaanalysis. Fourthly, two of the included studies were performed in Asia and one in Europe, thus reducing 
Appendix C - Cochrane Central Register of Controlled Trials (CENTRAL) search strategy <1996 to 22 May 2019>

1 exp Parenteral nutrition/

2 exp Parenteral nutrition, Home/

3 exp Parenteral nutrition, Total/

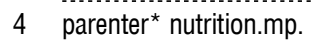

5 parenter* route.mp.

6 TPN.mp.

7 total nutrient admixture.mp.

8 central venous nutrition.mp.

9 parenter* feeding.mp.

10 nil per os.mp.

11 NPO.mp.

12 fasting.mp.

13 fluid therapy.mp.

14 intravenous feeding.mp

15 intravenous nutrition.mp.

16 (intravenous $^{*}$ near/2 feed ${ }^{\star}$ ).mp.

17 (intravenous* near/2 nutrition*).mp. $^{*}$

18 or $/ 1-17$

19 exp Enteral nutrition/

20 exp Eating/

21 oral feeding.mp.

22 oral diet.mp.

23 peroral diet.mp.

24 peroral feeding.mp.

25 enter $^{*}$ feeding.mp.

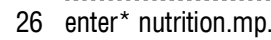

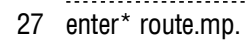

28 oral food intake.mp.

29 dietary intake.mp.

30 (feeding near/1 tube ${ }^{\star}$ ).mp.

31 gastric feeding.mp.

32 (jejunostomy and (tube ${ }^{*}$ or feed ${ }^{*}$ or tube feed $\left.\left.{ }^{*}\right)\right) \cdot m p$.

33 (nasojejunal and $\left(\right.$ tube ${ }^{\star}$ or feed ${ }^{\star}$ or tube feed $\left.{ }^{\star}\right)$ ).mp.

34 (nasogastric and $\left(\right.$ tube $^{*}$ or feed ${ }^{*}$ or tube feed $\left.\left.{ }^{*}\right)\right) \cdot \mathrm{mp}$.

35 (gastrojejunostomy and (tube ${ }^{\star}$ or feed ${ }^{\star}$ or tube feed $\left.{ }^{*}\right)$ ).mp

36 (percutaneous endoscopic gastrostomy and (tube ${ }^{\star}$ or feed ${ }^{\star}$ or tube feed $\left.\left.^{\star}\right)\right)$.mp.

37 (PEG and (tube ${ }^{\star}$ or line $\left.e^{\star}\right)$.mp

38 (percutaneous endoscopic jejunostomy and (tube $^{*}$ or feed ${ }^{*}$ or tube feed $\left.\left.^{*}\right)\right) \cdot \mathrm{mp}$

39 (JEJ and (tube* or line $\left.{ }^{*}\right)$ ).mp.

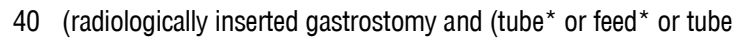
feed $\left.\left.^{\star}\right)\right)$.mp.

41 (RIG and (tube or line $\left.^{*}\right)$ ).mp.

42 (percutaneous transperitoneal jejunostomy and (tube ${ }^{\star}$ or feed ${ }^{\star}$ or tube feed $\left.\left.{ }^{\star}\right)\right) \cdot \mathrm{mp}$

generalizability to a North American context given the differences in approaches to postoperative convalescence (30). Lastly, the results of the present review do not extend to all types of pancreatic anastomosis (i.e., pancreaticogastrostomy), as all anastomosis performed in the included studies were pancreaticojejunostomies.
43 (percutaneous transperitoneal gastrojejunostomy and (tube ${ }^{*}$ or feed ${ }^{\star}$ or tube feed $\left.{ }^{*}\right)$.mp.

44 or $/ 19-43$

45 exp Nutritional support/

46 nutrition* support $^{*} \cdot \mathrm{mp}$

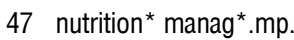

48 (nutrition* near/2 manag $\left.{ }^{\star}\right) \cdot \mathrm{mp}$.

49 artificial nutrition.mp.

50 artificial feeding.mp.

51 nutrition* $^{*}$ supplement* ${ }^{*} \mathrm{mp}$.

52 (nutrition* near/2 supplement*).mp

53 postoperative nutrition.mp.

54 perioperative nutrition.mp.

55 or $/ 45-54$

56 exp Fistula/

57 exp Digestive system fistula/

58 fistula*.mp.

59 fistuliz*.mp.

60 fistulis*.mp

61 leak*.mp.

62 or $/ 56-61$

63 exp Pancreatectomy/

64 pancreaticoduodenectomy/

65 pancreaticojejunostomy/

66 pancreatectomy.mp.

67 hemipancreatectomy.mp.

68 pancreaticoduodenectomy.mp

69 whipple.mp.

70 whipple procedure ${ }^{\star} . \mathrm{mp}$

71 whipple surgery.mp.

72 distal pancreaticoduodenectomy.mp.

73 pancreaticojejunostomy.mp.

74 pancrea* necrosectomy.mp.

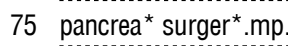

76 pancrea* procedure $^{\star} . \mathrm{mp}$.

77 pancrea* resection $^{\star} . \mathrm{mp}$.

78 (pancrea* near/2 surger $\left.{ }^{\star}\right) . m p$

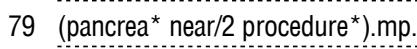

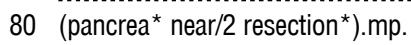

81 Pancreas/

82 pancrea*.mp.

83 or $/ 63-82$

84 (18 or 44 or 55 ) and 62 and 83

\section{CONCLUSION}

To our knowledge, this is the first systematic review and meta-analysis evaluating the type of nutritional supplementation on the management of POPF. We performed a rigorous review of the literature and 
Appendix D - Web of Science search strategy $<1900$ to 22 May 2019>

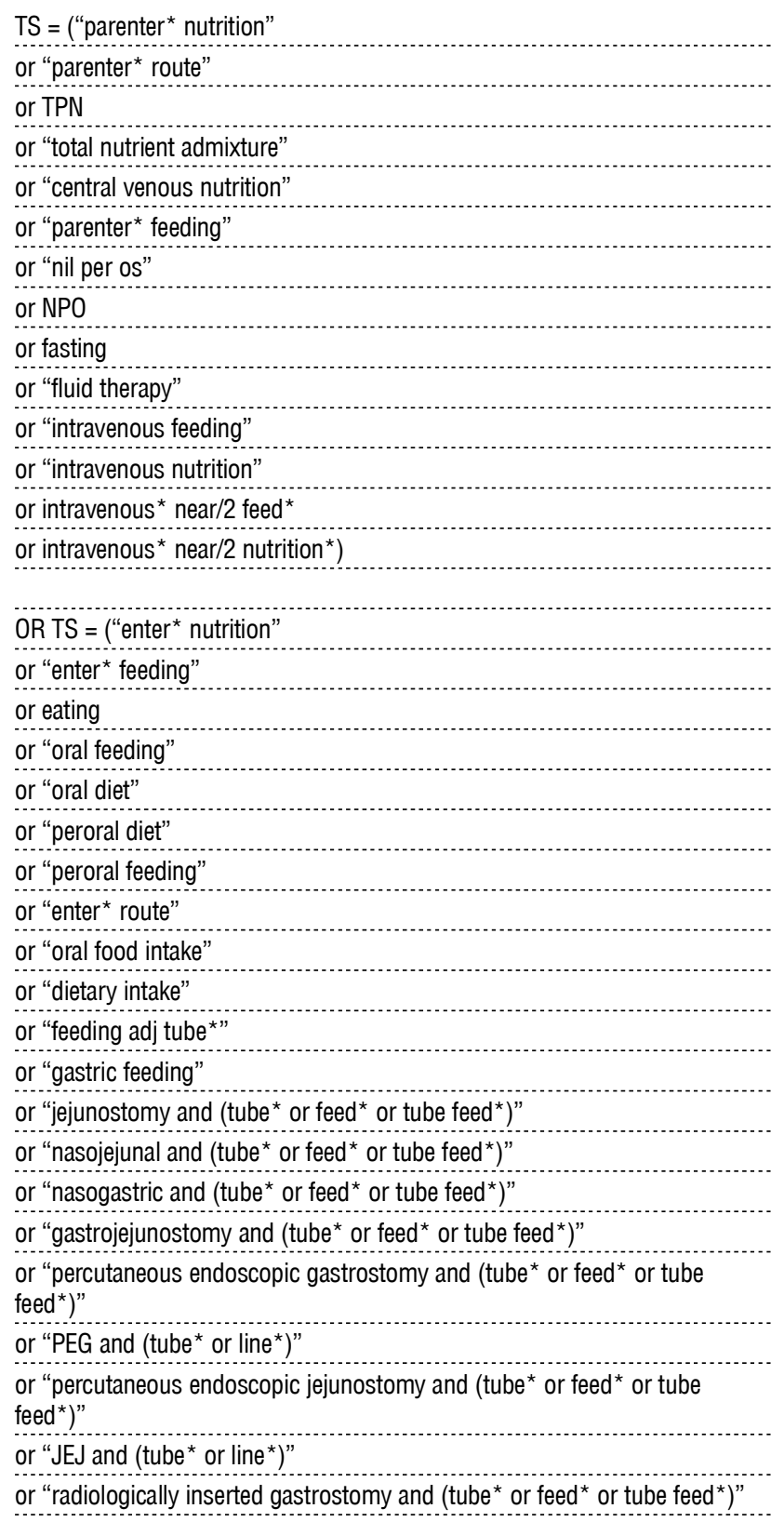

or "RIG and (tube* or line*)" or "percutaneous transperitoneal jejunostomy and (tube* or feed* or tube feed*)"

or "percutaneous transperitoneal gastrojejunostomy and (tube* or feed* or tube feed $\left.)^{\star \prime}\right)$

OR TS $=$ ("nutrition* support*"

or "nutrition* near/2 manag*"

or "artificial nutrition"

or "artificial feeding"

or "nutrition* near/2 supplement"

or "postoperative nutrition"

or "perioperative nutrition"

or "feeding method")

AND TS $=($ fistula *

or fistuliz*

or fistulis*

or leak $\left.{ }^{*}\right)$

AND TS = (pancreatectomy

or "distal pancreatectomy"

or "total pancreatectomy"

or hemipancreatectomy

or pancreaticoduodenectomy

or whipple

or "whipple procedure*"

or "whipple surgery"

or "distal pancreaticoduodenectomy"

or pancreaticojejunostomy

or "pancrea* necrosectomy"

or "pancrea* surger*"

or "pancrea* procedure*"

or "pancrea* resection *"

or "pancrea* near/2 surger ")

or "pancrea* near/2 procedure*"

or "pancrea* near/2 resection*"

or pancrea*) included only RCTs in order to decrease selection bias. Additionally, we evaluated the quality of evidence with GRADE to better inform clinicians on the certainty of our results. Overall, there was no data to suggest that the use of enteral nutrition is more effective than the use of parenteral nutrition at enhancing the rate of POPF closure. However, the quality of evidence that exists in the current literature is very low. Therefore, the decision between enteral and parenteral nutrition in this setting should still be based on physician preference and patient-specific factors. This study contributes to the current body of literature surrounding the management of POPF, as most previous study pertains to rate of POPF development as opposed to rate of POPF closure (31). Larger comparative studies including a wider range of pancreatic operations are required to confirm the findings of the present review and determine whether optimal management of POPF may differ based on index pancreatic operation. Furthermore, larger studies may allow for the examination of important secondary outcomes such as LOS and quality of life. Lastly, future study is required to reach consensus regarding the definition of fistula closure and other important secondary endpoints. 


\section{Funding}

This research did not receive any specific grant from funding agencies in the public, commercial, or notfor-profit sectors.

\section{Declaration of conflicting interests}

Dr. Serrano, Dr. Ruo, Dr. Doumouras, Ms. Tywonek, Dr. Lee, Ms. Lee, and Dr. McKechnie do not have proprietary or commercial interest in any product mentioned or concept discussed in this article to report.

\section{Author's contributions}

Study concept and design - Serrano, Ruo, Y. Lee, S. Lee. Acquisition of data - McKechnie, S. Lee. Analysis and interpretation of data - All authors. Drafting of the manuscript - All authors. Critical revision of the manuscript for intellectual content - All authors. Final approval of version to be published - All authors. Acceptance of public responsibility for the work - All authors.

\section{REFERENCES}

1. Diener MK, Seiler CM, Rossion I, Kleeff J, Glanemann M, Butturini $G$, et al. Efficacy of stapler versus hand-sewn closure after distal pancreatectomy (DISPACT): a randomised, controlled multicentre trial. Lancet. 2011;377(9776):1514-22.

2. Kleeff J, Diener MK, Z'Graggen K, Hinz U, Wagner M, Bachmann J, et al. Distal pancreatectomy: risk factors for surgical failure in 302 consecutive cases. Annals of surgery. 2007;245(4):573-82.

3. Knaebel HP, Diener MK, Wente MN, Buchler MW, Seiler CM. Systematic review and meta-analysis of technique for closure of the pancreatic remnant after distal pancreatectomy. The British journa of surgery. 2005;92(5):539-46.

4. Lillemoe KD, Kaushal S, Cameron JL, Sohn TA, Pitt HA, Yeo CJ. Distal pancreatectomy: indications and outcomes in 235 patients. Annals of surgery. 1999;229(5):693-8; discussion 8-700.

5. Bassi C, Marchegiani G, Dervenis C, Sarr M, Abu Hilal M, Adham M, et al. The 2016 update of the International Study Group (ISGPS) definition and grading of postoperative pancreatic fistula: 11 Years After. Surgery. 2017;161(3):584-91.

6. Veillette G, Dominguez I, Ferrone C, Thayer SP, McGrath D, Warshaw $\mathrm{AL}$, et al. Implications and management of pancreatic fistulas following pancreaticoduodenectomy: the Massachusetts General Hospital experience. Archives of surgery. 2008;143(5):476-81.

7. Malleo G, Pulvirenti A, Marchegiani G, Butturini G, Salvia R, Bassi C. Diagnosis and management of postoperative pancreatic fistula. Langenbecks Arch Surg. 2014;399(7):801-10.

8. Goonetilleke KS, Siriwardena AK. Systematic review of peri-operative nutritional supplementation in patients undergoing pancreaticoduodenectomy. JOP : Journal of the pancreas. 2006;7(1):5-13.

9. Wu JM, Kuo TC, Chen HA, Wu CH, Lai SR, Yang CY, et al Randomized trial of oral versus enteral feeding for patients with postoperative pancreatic fistula after pancreatoduodenectomy. British Journal of Surgery. 2019;106(3):190-8.

10. Gullo L, Priori P, Pezzilli R, Biliotti G, Mattioli G, Barbara L. Pancreatic secretory response to ordinary meals: studies with pure pancreatic juice. Gastroenterology. 1988;94(2):428-33.

11. O'Keefe SJ. Physiological response of the human pancreas to enteral and parenteral feeding. Curr Opin Clin Nutr Metab Care.
2006:9(5):622-8.

12. Blumenstein I, Shastri YM, Stein J. Gastroenteric tube feeding: techniques, problems and solutions. World journal of gastroenterology: WJG. 2014;20(26):8505-24.

13. Zeki S, Culkin A, Gabe SM, Nightingale JM. Refeeding hypophosphataemia is more common in enteral than parenteral feeding in adult in patients. Clinical nutrition. 2011;30(3):365-8.

14. Bassi C, Dervenis C, Butturini G, Fingerhut A, Yeo C, lzbicki J, et al. Postoperative pancreatic fistula: an international study group (ISGPF) definition. Surgery. 2005;138(1):8-13.

15. Munoz-Bongrand N, Sauvanet A, Denys A, Sibert A, Vilgrain V, Belghiti J. Conservative management of pancreatic fistula after pancreaticoduodenectomy with pancreaticogastrostomy. Journal of the American College of Surgeons. 2004;199(2):198-203.

16. Pannegeon V, Pessaux P, Sauvanet A, Vullierme MP, Kianmanesh R, Belghiti J. Pancreatic fistula after distal pancreatectomy - Predictive risk factors and value of conservative treatment. Archives of surgery. 2006;141(11):1071-6.

17. Fan BG. Effects of parenteral nutrition on the exocrine pancreas in response to cholecystokinin. Journal of Parenteral and Enteral Nutrition. 2008;32(1):57-62.

18. Fong YM, Marano MA, Barber A, He W, Moldawer LL, Bushman ED, et al. Total Parenteral-Nutrition and Bowel Rest Modify the Metabolic Response to Endotoxin in Humans. Annals of surgery. 1989; 210(4):449-57.

19. Moher D, Liberati A, Tetzlaff J, Altman DG, Group P. Preferred reporting items for systematic reviews and meta-analyses: the PRISMA statement. Annals of internal medicine. 2009;151(4):264-9, W64.

20. Landis JR, Koch GG. The measurement of observer agreement for categorical data. Biometrics. 1977;33(1):159-74.

21. Wan X, Wang WQ, Liu JM, Tong TJ. Estimating the sample mean and standard deviation from the sample size, median, range and/or interquartile range. Bmc Med Res Methodol. 2014;14.

22. Higgins JPT, Altman DG, Gotzsche PC, Juni P, Moher D, Oxman AD, et al. The Cochrane Collaboration's tool for assessing risk of bias in randomised trials. Bmj-Brit Med J. 2011;343.

23. Guyatt GH, Oxman AD, Vist GE, Kunz R, Falck-Ytter Y, Alonso-Coello $P$, et al. GRADE: an emerging consensus on rating quality of evidence and strength of recommendations. Br Med J. 2008; 336(7650):924-6

24. Klek S, Sierzega M, Turczynowski L, Szybinski P, Szczepanek K, Kulig J. Enteral and parenteral nutrition in the conservative treatment of pancreatic fistula: a randomized clinical trial. Gastroenterology. 2011;141(1):157-63, 63 e1.

25. Fujii T, Yamada S, Murotani K, Okamura Y, Ishigure K, Kanda M, et al. Oral Food Intake Versus Fasting on Postoperative Pancreatic Fistula After Distal Pancreatectomy: A Multi-Institutional Randomized Controlled Trial. Medicine (Baltimore). 2015;94(52): e2398.

26. Fujii T, Nakao A, Murotani K, Okamura Y, Ishigure K, Hatsuno T, et al. Influence of Food Intake on the Healing Process of Postoperative Pancreatic Fistula After Pancreatoduodenectomy: A Multi-institutional Randomized Controlled Trial. Annals of surgical oncology. 2015;22(12):3905-12.

27. Gaujoux S, Cortes A, Couvelard A, Noullet S, Clavel L, Rebours V, et al. Fatty pancreas and increased body mass index are risk factors of pancreatic fistula after pancreaticoduodenectomy. Surgery. 2010; 148(1):15-23.

28. Nahm CB, Connor SJ, Samra JS, Mittal A. Postoperative pancreatic fistula: a review of traditional and emerging concepts. Clin Exp Gastroenterol 2018:11:105-18.

29. Nishida $\mathrm{Y}$, Kato $\mathrm{Y}$, Kudo M, Aizawa H, Okubo S, Takahashi D, et al. Preoperative Sarcopenia Strongly Influences the Risk of Postoperative Pancreatic Fistula Formation After Pancreaticoduodenectomy. Journal of gastrointestinal surgery : official journal of the Society for Surgery of the Alimentary Tract. 2016;20(9):1586-94.

30. Fong ZV, Tanabe KK. The clinical management of hepatocellular carcinoma in the United States, Europe, and Asia: A comprehensive and evidence-based comparison and review. Cancer. 2014;120(18): 2824-2838.

31. Adiamah A, Ranat R, Gomez D. Enteral versus parenteral nutrition following pancreaticoduodenectomy: a systematic review and metaanalysis. HPB (Oxford). 2019;21(7):793-801. 